\title{
Role of Maspin, CK17 and Ki-67 Immunophenotyping in Diagnosing of Pancreatic Ductal Adenocarcinoma in Endoscopic Ultrasound-Guided Fine Needle Aspiration Cytology
}

\author{
Mona M Mamdouh ${ }^{1 *}$, Hussein Okasha ${ }^{2}$, Hebat Allah M Shaaban ${ }^{1}$, Nesreen H \\ Hafez $^{1}$, Emad Hamza El-Gemeie ${ }^{1}$
}

\begin{abstract}
Background and study aim: One of the problems in diagnosing pancreatic ductal adenocarcinoma (PDAC) is differentiation between PDAC cells and benign pancreatic tissue cells in cytologic samples. This study aimed to evaluate the usefulness of Maspin, CK17 and Ki-67 immunocytochemistry (ICC) in differentiation between these two groups of cells. Materials and methods: This retrospective study was carried on 80 cases of PDAC and 25 cell blocks of benign pancreatic tissue cells as a control group for evaluation of Maspin, CK17 and Ki-67 ICC. PDAC cases were sampled by endoscopic ultrasound guided fine needle aspiration cytology (EUS-FNAC), while cell blocks of control group were aspirated from benign pancreatic tissues that were obtained from the pancreatic surgically resected specimens. Immunostaining patterns, sensitivity, specificity, positive predictive value (PPV), negative predictive value (NPV) and accuracy of each antibody as well as possible antibody combined panels of these markers in differentiation between the two groups were evaluated. Results: Positive immunoreactivity for Maspin, CK17 and Ki-67 were 92.5\%, 80\% and $72.5 \%$ in PDAC cases, respectively. In contrast to PDAC cases, all the cell blocks of benign pancreatic tissue cells were negative for these markers. Regarding different panels, combined use of Maspin, CK17 and Ki-67 together as a triple test (at least one of them is positive) achieved the highest sensitivity of $98.8 \%$, specificity of $100 \%$, PPV of $100 \%$, NPV of $96.2 \%$ and accuracy of $99 \%$ in the differentiation between PDAC and benign pancreatic tissue. Conclusion: Employing this short panel [Maspin, CK17 and Ki-67] is helpful for better differentiation between PDAC and benign pancreatic tissue.
\end{abstract}

Keywords: Pancreatic ductal adenocarcinoma- EUS-FNAC- Maspin- CK17- Ki-67

Asian Pac J Cancer Prev, 22 (10), 3299-3307

\section{Introduction}

Pancreatic ductal adenocarcinoma (PDAC) accounts for nearly $85 \%$ of solid pancreatic tumors (Reid and Centeno, 2014; Aksoy-Altinboga et al., 2018). PDAC is the fourth leading cause of cancer-related death in the world (Foucher et al., 2018). Approximately 80\% of patients with pancreatic cancer have unresectable disease at diagnosis because of early loco-regional extension or distant metastases (He et al., 2014). Recent studies reported the potentiality of neoadjuvant therapy to decrease the size of tumors and make them more resectable, therefore a definite preoperative diagnosis of PDAC is important for all patients (Furuhata et al., 2017). Endoscopic ultrasound (EUS) is one of the best methods for detecting pancreatic cancer (Dimastromatteo et al., 2017). In addition, endoscopic ultrasound-guided fine needle aspiration cytology (EUS-FNAC) for pancreatic lesions is a safe and efficient procedure and has become a popular approach for obtaining diagnostic pancreatic samples (Kudo et al., 2014).

The EUS-FNAC is a well-established method to accurately diagnose high-grade PDAC, while interpretation of low grade PDAC can be challenging to be differentiated from benign pancreatic tissue (Dim et al., 2014; Furuhata et al., 2017), even in tissue biopsies (Liu et al., 2012). Application of appropriate immunocytochemistry (ICC) markers on cell blocks enables cytopathologists to differentiate PDAC from benign mimickers (Lin et al., 2015; Furuhata et al., 2017). A growing number of potential immunostaining markers in diagnosing PDAC have emerged, however reports that have specifically focused on distinguishing PDAC from benign mimickers, especially in FNAC, are very limited (Furuhata et al., 2017). Some studies showed that expression of Maspin (Cao et al., 2007; Liu et al., 2012;

${ }^{1}$ Department of Pathology, National Cancer Institute, Cairo University, Cairo, Egypt. ${ }^{2}$ Department of Internal Medicine, Gastroenterology and Hepatology division, Kasr Al- Aini Hospitals, Cairo University, Cairo, Egypt. *For Correspondence: dr.mona.mamdouh.nci@gmail.com 
Berardi et al., 2013; Lok et al., 2014; Furuhata et al., 2017; Aksoy-Altinboga et al,. 2018), CK17 (Goldstein and Bassi, 2001; Chu et al., 2005; Sarbia et al., 2007; Yang et al., 2012; Berardi et al., 2013) and Ki-67 (Klein et al., 2002; Jahng et al., 2010; Karamitopoulou et al., 2010; Kim et al., 2015) are conspicuous for diagnosing PDAC.

Maspin is a unique member of the serpin superfamily of serine proteinase inhibitors and is located on chromosome 18q21.3-q23. Maspin was originally described as a tumor suppressor gene inhibiting cell motility, invasiveness and metastases (Umekita et al., 2006). However, the correlation between Maspin immunostaining expression and worse prognoses was reported in many cancers (Umekita et al., 2002; Hirai et al., 2005). Maspin was documented to be overexpressed in PDAC in both tissue biopsies and cell blocks obtained by FNAC (Cao et al., 2007; Liu et al., 2012; Berardi et al., 2013; Lok et al., 2014; Furuhata et al., 2017; Aksoy-Altinboga et al., 2018).

CK17 is a low-molecular-weight keratin that is normally expressed in myoepithelial, basal cells and subsets of hair shaft epithelia (Chu et al., 2005; Lok et al., 2014). It is also expressed mainly in squamous, basal and transitional cell carcinomas as well as in adenocarcinomas with squamous differentiation (Goldstein and Bassi, 2001; Yang et al., 2012). Few studies had shown that CK17 also might be a useful marker for the diagnosis of pancreaticobiliary adenocarcinomas (Goldstein and Bassi, 2001; Berardi et al., 2013) and separating them from extra-pancreaticobiliary non-mucinous adenocarcinomas (Chu et al., 2005; Sarbia et al., 2007; Yang et al., 2012).

$\mathrm{Ki}-67$ is a nuclear protein that is increased in proliferating cells and present in all phases of the cell cycle, except the resting phase (Jahng et al., 2010). Overexpression is frequently seen in a variety of malignant tumors and is associated with worse prognosis (Stuart-Harris et al., 2008; Viale et al., 2008). A stepwise progression from normal pancreatic epithelium to pancreatic intraepithelial neoplasia (PanIN) and then to frank adenocarcinoma has been correlated with increasing Ki-67 labeling index expression (Klein et al., 2002; Jahng et al., 2010; Karamitopoulou et al., 2010; Kim et al., 2005; Furuhata et al., 2017).

Therefore, this study aimed to evaluate the usefulness of Maspin, CK17 and Ki-67 ICC as separate markers and as different combined panels in differentiation between PDAC cells and benign pancreatic tissue cells.

\section{Materials and Methods}

This retrospective study was conducted on 80 cases of PDAC and 25 cell blocks of benign pancreatic tissue cells as a control group in National Cancer Institute (NCI), Cairo University.

\section{EUS-FNAC procedure and preparation of the cases}

The aforementioned 80 cases were aspirated by EUS-FNAC that was performed using 22-gauge needle with rapid-on-site evaluation (ROSE) in the Endoscopic Unit. Smears were prepared from the aspirate of the needle pass: at least, one was air-dried and Diff-Quik-stained (used for ROSE) and the other was fixed in 95\%
Ethyl alcohol for minutes at room temperature. If the obtained sample was non-diagnostic, additional passes were attempted until diagnostic material is obtained. Additional passes or needle rinse material was collected in solution [10\% neutral-buffered formalin: alcohol, 1:9] for subsequent preparation of cell block. In addition, some material was collected in SurePath liquid based cytology (LBC) preservative.

All the aspirated material was sent to the Cytology Unit, for preparation, immunostaining and cytological diagnosis. The Ethyl alcohol-fixed slides were stained using Papanicolaou stain, while cell block material was prepared and stained with HandE stain. Whereas Surepath LBC material was prepared and stained using SurePath LBC apparatus.

Regarding the 25 cell blocks of control group, they were prepared from aspirated benign pancreatic tissues that were obtained from the pancreatic surgically resected specimens that were sent to our Pathology Unit (25 Whipple operations for PDAC) and then stained using HandE stain.

\section{Selection of cases}

These PDAC cases were retrieved from the database of Cytology Unit, during the period from January 2014 to December 2019. The selected criteria of the cases included unequivocal cytologic reports of PDAC, availability of all studied correlated clinical and radiologic/endoscopic data, availability of formalin-fixed paraffin-embedded cell blocks with adequate tumor cells to perform ICC and no administered chemo- or radiotherapy before FNAC.

\section{Cytologic examination and interpretation}

The review of diagnosis and grading of our cases was done according to "The Papanicolaou Society of Cytopathology System for Reporting Pancreaticobiliary Cytology" (Pitman et al., 2014). The diagnosis of the cases was correlated with their final histopathological diagnoses (if present) and radiologic/endoscopic data that included malignant criteria of the pancreatic mass, pathological lymph nodes and/or presence of distant metastases. Also clinical course was considered.

\section{Immunocytochemical (ICC) staining}

For immunostaining, Paraffin embedded sections of cell blocks of PDAC cases and the control group were made at 4 microns thickness and mounted on positive charged slides. Immunostaining was done by BenchMark IHC/ISH staining module (Ventana immunostaining system), the following steps occurred automatically: 1) Deparaffinization, 2) Cell conditioning (Standard CC1 application) for 80 minutes, 3) Application of one drop $(100 \mu)$ of the antibody, 4) Application of cover slip and incubation for 32 minutes, 5) Application of one drop of DAB (counterstain) with Haematoxylin II and incubation for 8 minutes, 6) Application of Bluing Reagent for 4 minutes, 7) Slides were extracted and arranged in racks, 8) Slides were washed in tap water and soap for 5 minutes and then dehydrated in the ascending grades of alcohol for 5 minutes in each container, 9) Slides were cleared in xyline, and then cover slips were applied. 
Appropriate positive and negative controls were included in each run. The selected markers were Maspin (clone BSB-92, Ready to use, Gene Tex), CK17 (clone E3, Ready to use, Dako) and Ki-67 (clone MIB-1, Ready to use, Dako).

Immunocytochemical evaluation, scoring and interpretation

ICC evaluation was done, including assessment of subcellular immunostaining localization, the percentage of positively stained cells and the immunostaining intensity. Nuclear and/or cytoplasmic immunostaining for Maspin, cytoplasmic immunostaining for CK17 and nuclear immunostaining for $\mathrm{Ki}-67$ were considered as a definite positivity. The percentage of immunostained tumor cells was recorded as negative $(<5 \%$ of tumor cells stained $), 1+(5 \%-25 \%), 2+(26 \%-50 \%), 3+$ $(51 \%-75 \%)$, and $4+(>75 \%)$, hence the cutoff point of positivity for all studied markers is 5\% (Chu et al., 2005; Aksoy-Altinboga et al., 2018). Scores $1+$ and 2+ were considered "focal immunostaining", while scores $3+$ and $4+$ were considered "diffuse immunostaining" (Chu et al., 2005; Cao et al., 2007; Liu et al., 2012; Lok et al., 2014). The immunostaining intensity was graded as: no immunostaining, weak immunostaining, moderate immunostaining and strong immunostaining (Goldstein and Bassi, 2001; Karamitopoulou et al., 2010; Yang et al., 2012; Lok et al., 2014).

We correlated immunostaining results of PDAC cases with some clinico-pathological data.

\section{Statistical analysis}

Data management and analysis were performed using Statistical Package for Social Sciences (SPSS) vs. 25 . Numerical data were summarized using means and standard deviations or medians and/or ranges, as appropriate. Categorical data were summarized as numbers and percentages. Numerical data were explored for normality using Kolmogrov-Smirnov test and ShapiroWilk test. Chi square or Fisher's tests was used to compare between independent groups with respect to categorical data. Comparisons between two groups for normally distributed numeric variables were done using the Student's t-test while for non-normally distributed numeric variables, comparisons were done by Mann-Whitney U test. To measure the strength of association between the non-normally distributed measurements, Spearman's correlation coefficients was calculated ( $\mathrm{r}$ is the correlation coefficient, it ranges from -1 to +1$),(+1$ indicates positive correlation, -1 indicates negative correlation, 0 indicates no correlation). Receiver operating characteristic curve (ROC curve) was done to determine the best sensitivity, specificity and area under the curve. The accuracy of the test depends on how well the test separates the group being tested into those with and without the disease in question. All tests were two tailed and Probability ( $p$-value) $\leq 0.05$ is considered significant.

\section{Results}

Our PDAC cases included 51 male and 29 female with male: female ratio of 1.8:1 and a mean age of 60 years. Among the studied cases, recorded reports of CA19-9 serum level were only available for 63 cases and those of CEA serum level were available for 58 cases. High CA199 and CEA serum levels were documented in $74.6 \%$ and $37.9 \%$ of available reports, respectively. The majority of our cases were located at the head of the pancreas, represented $71.2 \%$. Regarding TNM staging groups, the majority of our cases were of high stage; stage III and

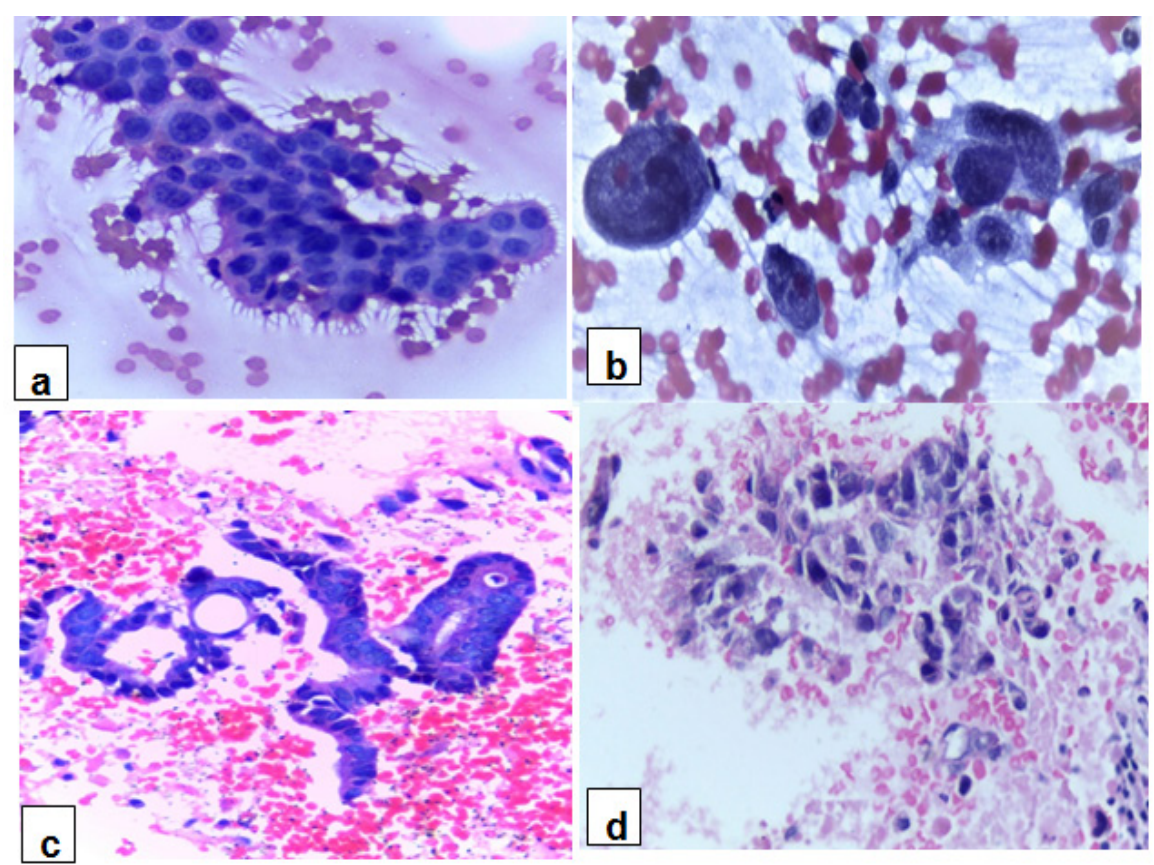

Figure 1. (a\&b) cytologic smears of PDAC, showing nuclear enlargement, nuclear membrane irregularity and anisonucleosis: (a) Malignant ductal epithelial cells, arranged in an uneven, "drunken honeycomb" sheet (Papanicolaoustained $\mathrm{x} 40$ ) and (b) loosely cohesive syncytial tissue fragments, with some separate forms (Papanicolaou-stained $\mathrm{x} 40$ ). (c and d) cell block sections of PDAC: (c) Irregular and angulated tubules and (d) loosely cohesive sheets $($ H\&E-stained $\times 40)$. 
Table 1. Clinico-Pathological Data of Our 80 Cases of PDAC

\begin{tabular}{|c|c|c|c|}
\hline Criterion & & Variables & $\mathrm{n}(\%)$ \\
\hline \multirow[t]{2}{*}{$\operatorname{Sex}(n=80)$} & & Female & $29(36.2)$ \\
\hline & & Male & $51(63.8)$ \\
\hline \multirow[t]{2}{*}{ Age $(n=80)$} & & Mean \pm SD & $60 \pm 10$ \\
\hline & & Median (range) & $59(28-77)$ \\
\hline \multicolumn{4}{|c|}{ Serum markers* } \\
\hline & \multirow{2}{*}{$\begin{array}{l}\text { CA19-9 } \\
(\mathrm{n}=63)\end{array}$} & High & 47 (74.6) \\
\hline & & Normal & $16(25.4)$ \\
\hline & \multirow{2}{*}{$\begin{array}{l}\text { CEA } \\
(\mathrm{n}=58)\end{array}$} & High & $22(37.9)$ \\
\hline & & Normal & $36(62.1)$ \\
\hline \multirow[t]{5}{*}{ Site $(\mathrm{n}=80)$} & & Head & $57(71.2)$ \\
\hline & & Body & $6(7.5)$ \\
\hline & & Head \& body & $5(6.3)$ \\
\hline & & Body \& tail & $5(6.3)$ \\
\hline & & Tail & $7(8.7)$ \\
\hline \multicolumn{4}{|c|}{ TNM staging** } \\
\hline & \multirow[t]{4}{*}{ T-stage } & $\mathrm{T} 1$ & $2(2.5)$ \\
\hline & & $\mathrm{T} 2$ & $7(8.7)$ \\
\hline & & $\mathrm{T} 3$ & $46(57.5)$ \\
\hline & & $\mathrm{T} 4$ & $25(31.3)$ \\
\hline & \multirow[t]{2}{*}{$\mathrm{N}$-stage } & No & $27(33.7)$ \\
\hline & & N1 & $53(66.3)$ \\
\hline & \multirow[t]{2}{*}{ M-stage } & M0 & $45(56.2)$ \\
\hline & & M1 & $35(43.8)$ \\
\hline & \multirow{5}{*}{$\begin{array}{l}\text { Staging } \\
\text { group }\end{array}$} & I & $2(2.5)$ \\
\hline & & IIA & $8(10)$ \\
\hline & & IIB & $23(28.7)$ \\
\hline & & III & $12(15)$ \\
\hline & & IV & $35(43.8)$ \\
\hline \multirow[t]{3}{*}{ Grade } & & Well & $5(6.3)$ \\
\hline & & Moderate & $51(63.7)$ \\
\hline & & $\begin{array}{l}\text { Poorly } \\
\text { differentiated }\end{array}$ & $24(30)$ \\
\hline
\end{tabular}

*, NCI normal references for CA19-9 \& CEA; 0-22.3 U/ml and 0-5 ng/ml, respectively; **, TNM staging according to AJCC/UICC (Cong et al., 2018)

IV represented $15 \%$ and $43.8 \%$, respectively. Most of cases were moderately differentiated tumors, represented $63.7 \%$ (Table 1).

\section{Immunocytochemical findings}

The immunostaining results for PDAC cases are summarized in (Table 2). Out of the studied 80 PDAC cases, $74(92.5 \%)$ were positive for Maspin, with the majority showed strong intensity, represented $90.54 \%$. Among Maspin positive PDAC cases, 57 cases (77\%) showed both nuclear and cytoplasmic immunostaining, while 16 cases $(21.6 \%)$ showed nuclear immunostaining and only 1 case (1.4\%) showed cytoplasmic immunostaining. CK17 was positive in 64 out of 80 PDAC cases (80\%), with strong intensity in the majority of positive cases, represented $90.63 \%$. Ki-67 expression was noted in 58 (72.5\%) out of 80 studied PDAC cases. Regarding Ki-67

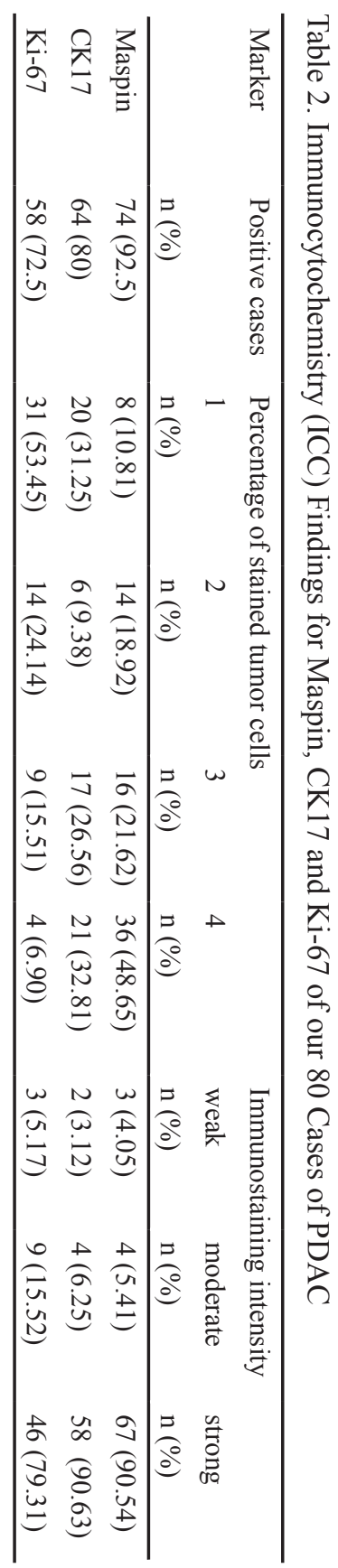

Table 3. The Sensitivity, Specificity, PPV, NPV and Accuracy of Maspin, CK17 and Ki-67 ImmunocytoChemistry (ICC), each one Individually and as Different Panels

\begin{tabular}{lccccc}
\hline ICC markers & $\begin{array}{c}\text { Sensitivity } \\
(\%)\end{array}$ & $\begin{array}{c}\text { Specificity } \\
(\%)\end{array}$ & $\begin{array}{c}\text { PPV } \\
(\%)\end{array}$ & $\begin{array}{c}\text { NPV } \\
(\%)\end{array}$ & $\begin{array}{c}\text { Accuracy } \\
(\%)\end{array}$ \\
\hline Maspin & 92.5 & 100 & 100 & 80.6 & 94.2 \\
CK17 & 80 & 100 & 100 & 61 & 84.8 \\
Ki-67 & 72.5 & 100 & 100 & 53.2 & 79 \\
$\begin{array}{l}\text { Maspin/CK17/ } \\
\text { Ki-67 panel* }\end{array}$ & 98.8 & 100 & 100 & 96.2 & 99 \\
$\begin{array}{l}\text { Maspin/CK17 } \\
\text { panel* }\end{array}$ & 97.5 & 100 & 100 & 92.6 & 98.1 \\
$\begin{array}{l}\text { Maspin/Ki-67 } \\
\text { panel* }\end{array}$ & 95 & 100 & 100 & 86.2 & 96.2 \\
$\begin{array}{l}\text { CK17/Ki-67 } \\
\text { panel* }\end{array}$ & 92.5 & 100 & 100 & 80.6 & 94.3 \\
\hline
\end{tabular}

PPV, Positive Predictive Value ; NPV, Negative Predictive Value; *, The test was considered positive if any of the markers was positive. 


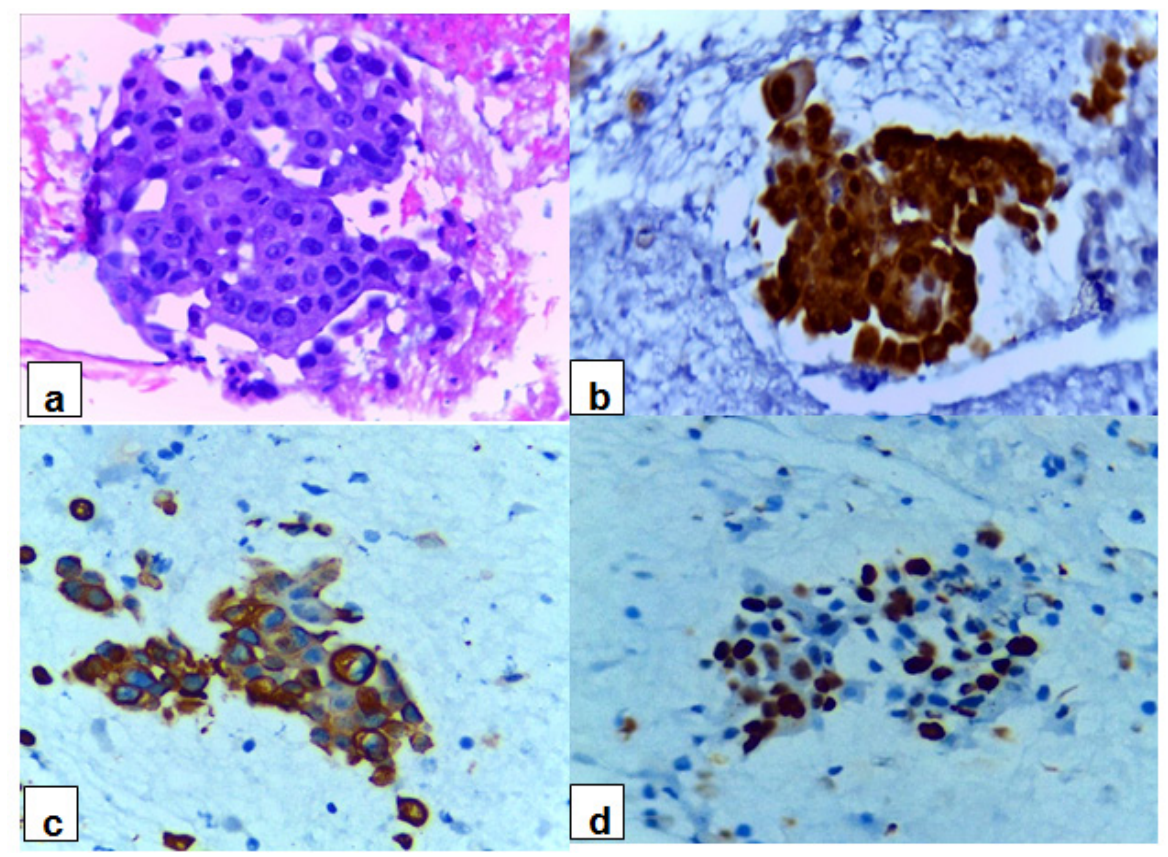

Figure 2. A Case of PDAC Reveals Malignant Pancreatic Ductal Cells: (a) cell block section (H\&E-stained x40), (b) Maspin shows diffuse positive nuclear and cytoplasmic immunostaining for malignant cells with strong intensity (x40), (c) CK17 shows diffuse positive cytoplasmic immunostaining for malignant cells with strong intensity (x40) and (d) Ki-67 shows diffuse positive nuclear immunostaining for malignant cells with strong intensity (x40).

immunostaining intensity, the majority of positive PDAC cases showed strong intensity, represented $79.31 \%$. The majority of positive PDAC cases for Maspin and CK17 exhibited diffuse immunostaining, represented $70.27 \%$ and $59.37 \%$, respectively, whereas Ki-67 showed focal immnunostaining in $77.59 \%$ of the cases (Figure 2 and 3 ).

In contrast to PDAC cases, all the cell blocks of benign pancreatic tissue cells were negative for Maspin, CK17 and Ki-67 (Figure 4), with statistical significant difference in differentiation between the two groups $(\mathrm{P}<0.01)$.
In differentiation of PDAC from benign pancreatic tissue, Maspin showed sensitivity of $92.5 \%$, NPV of $80.6 \%$ and accuracy of $94.2 \%$. CK17 sensitivity, NPV and accuracy were of $80 \%, 61 \%$ and $84.8 \%$, respectively. Finally, Ki-67 had sensitivity of $72.5 \%$, NPV of $53.2 \%$ and accuracy of $79 \%$. Specificity and PPV were $100 \%$ for each marker. Regarding different panels, combined use of the three markers together as a triple test (at least one of them is positive) achieved the highest sensitivity of $98.8 \%$, NPV of $96.2 \%$ and accuracy of $99 \%$ in the

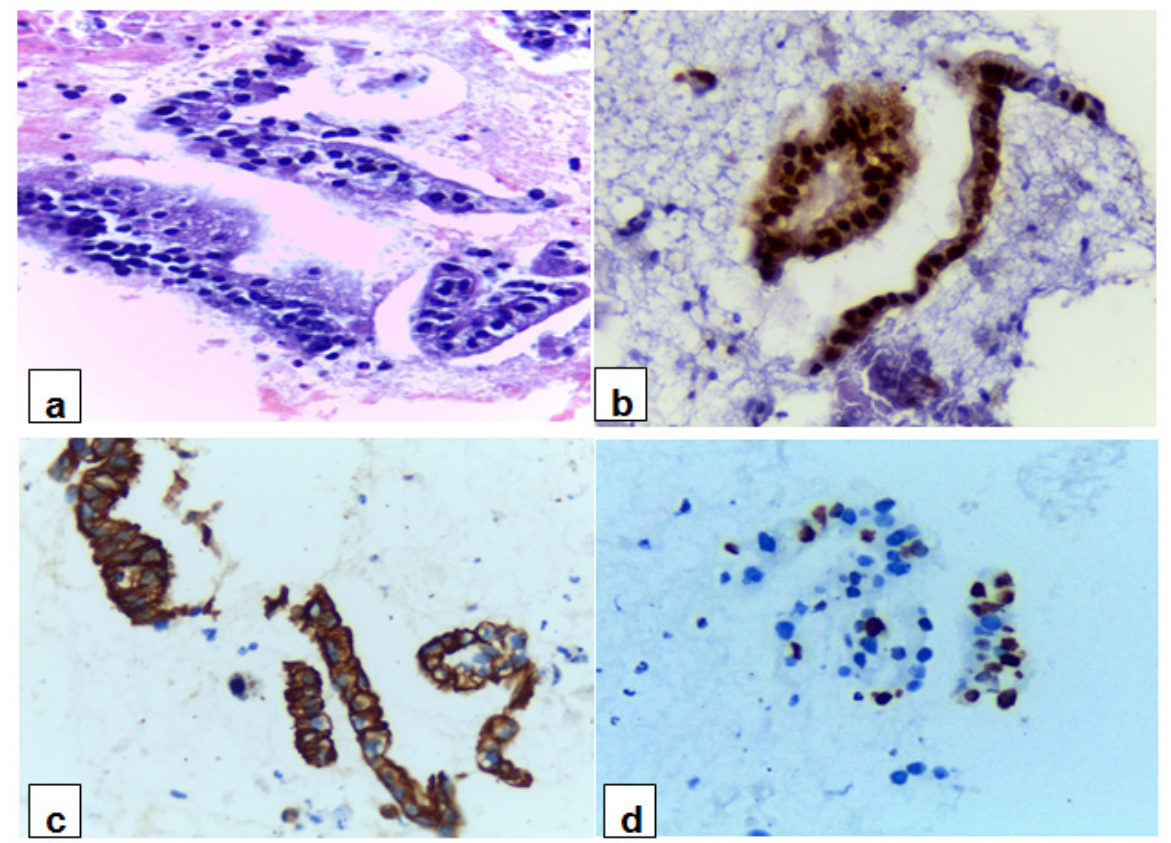

Figure 3. A cases of PDAC Reveals Malignant Pancreatic Ductal Cells: (a) cell block section (H\&E-stained x40), (b) Maspin shows diffuse positive nuclear and cytoplasmic immunostaining for malignant cells with strong intensity (x40), (c) CK17 shows diffuse positive cytoplasmic immunostaining for malignant cells with strong intensity (x40) and (d) Ki-67 shows focal positive nuclear immunostaining for malignant cells with strong intensity (x40). 
Table 4. Relationship between Clinico-Pathological Data of PDAC Cases and Studied Markers

\begin{tabular}{|c|c|c|c|c|c|c|c|c|c|}
\hline \multirow{2}{*}{$\begin{array}{l}\text { Clinico-pathologic } \\
\text { variables }\end{array}$} & \multicolumn{3}{|c|}{ Mapsin } & \multicolumn{3}{|c|}{ CK17 } & \multicolumn{3}{|c|}{ Ki67 } \\
\hline & $\begin{array}{l}\text { Positive } \\
\text { n (\%) }\end{array}$ & $\begin{array}{c}\text { Negative } \\
\mathrm{n}(\%)\end{array}$ & $\mathrm{p}$ & $\begin{array}{l}\text { Positive } \\
\text { n (\%) }\end{array}$ & $\begin{array}{c}\text { Negative } \\
\mathrm{n}(\%)\end{array}$ & $\mathrm{p}$ & $\begin{array}{l}\text { Positive } \\
\text { n (\%) }\end{array}$ & $\begin{array}{c}\text { Negative } \\
\mathrm{n}(\%)\end{array}$ & $\mathrm{p}$ \\
\hline \multicolumn{10}{|l|}{ Age $(n=80)$} \\
\hline Mean \pm SD & $60 \pm 10$ & $61 \pm 8$ & 0.862 & $61 \pm 10$ & $56 \pm 9$ & 0.116 & $60 \pm 9$ & $59 \pm 11$ & 0.592 \\
\hline Median (range) & $59(28-77)$ & $58(54-73)$ & & $61(28-77)$ & $56(36-72)$ & & $60(43-75)$ & $59(28-$ & 77) \\
\hline \multicolumn{10}{|l|}{$\operatorname{Sex}(n=80)$} \\
\hline Female & $28(96.6)$ & $1(3.4)$ & 0.409 & $24(82.8)$ & $5(17.2)$ & 0.775 & $25(86.2)$ & $4(13.8)$ & 0.067 \\
\hline Male & $46(90.2)$ & $5(9.8)$ & & $40(78.4)$ & $11(21.6)$ & & $33(64.7)$ & $18(35.3)$ & \\
\hline \multicolumn{10}{|l|}{ CA19-9 $(n=63)$} \\
\hline High & $43(91.5)$ & $4(8.5)$ & 1 & $40(85.1)$ & $7(14.9)$ & 0.448 & $32(68.1)$ & $15(31.9)$ & 0.757 \\
\hline Normal & $15(93.8)$ & $1(6.2)$ & & $12(75)$ & $4(25)$ & & $12(75)$ & $4(25)$ & \\
\hline \multicolumn{10}{|l|}{ CEA $(n=58)$} \\
\hline High & $20(90.9)$ & $2(9.1)$ & 0.63 & $20(90.9)$ & $2(9.1)$ & 0.088 & $15(68.2)$ & $7(31.8)$ & 0.35 \\
\hline Normal & $34(94.4)$ & $2(5.6)$ & & $26(72.2)$ & $10(27.8)$ & & $29(80.6)$ & $7(19.4)$ & \\
\hline \multicolumn{10}{|l|}{ Anatomical site $(\mathrm{n}=80)$} \\
\hline Head & $51(89.5)$ & $6(10.5)$ & NA & 45 (78.9) & $12(21.1)$ & NA & $43(75.4)$ & $14(24.6)$ & NA \\
\hline Body & $6(100)$ & $0(0)$ & & $6(100)$ & $0(0)$ & & $2(33.3)$ & $4(66.7)$ & \\
\hline Head\& body & $5(100)$ & $0(0)$ & & $4(80)$ & $1(20)$ & & $5(100)$ & $0(0)$ & \\
\hline Body\& tail & $5(100)$ & $0(0)$ & & $4(80)$ & $1(20)$ & & $3(60)$ & $2(40)$ & \\
\hline Tail & $7(100)$ & $0(0)$ & & $5(71.4)$ & $2(28.6)$ & & $5(71.4)$ & $2(28.6)$ & \\
\hline \multicolumn{10}{|l|}{ T stage $(\mathrm{n}=80)$} \\
\hline $\mathrm{T} 1$ & $2(100)$ & $0(0)$ & NA & $2(100)$ & $0(0)$ & NA & $2(100)$ & $0(0)$ & NA \\
\hline $\mathrm{T} 2$ & $7(100)$ & $0(0)$ & & $6(85.7)$ & $1(14.3)$ & & $5(71.4)$ & $2(28.6)$ & \\
\hline $\mathrm{T} 3$ & $41(89.1)$ & $5(10.9)$ & & 33 (71.7) & $13(28.3)$ & & $31(67.4)$ & $15(32.6)$ & \\
\hline $\mathrm{T} 4$ & $24(96)$ & $1(4)$ & & $23(92)$ & $2(8)$ & & $20(80)$ & $5(20)$ & \\
\hline \multicolumn{10}{|l|}{$\mathrm{N}$ stage $(\mathrm{n}=80)$} \\
\hline No & $25(92.6)$ & $2(7.4)$ & 1 & $22(81.5)$ & $5(18.5)$ & 0.813 & $20(74.1)$ & $7(25.9)$ & 0.822 \\
\hline N1 & $49(92.5)$ & $4(7.5)$ & & $42(79.2)$ & $11(20.8)$ & & $38(71.7)$ & $15(28.3)$ & \\
\hline \multicolumn{10}{|l|}{ M stage $(n=80)$} \\
\hline M0 & $41(91.1)$ & $4(8.9)$ & 0.691 & $35(77.8)$ & $10(22.2)$ & 0.779 & $32(71.1)$ & $13(28.9)$ & 0.805 \\
\hline M1 & 33 (94.3) & $2(5.7)$ & & $29(82.9)$ & $6(17.1)$ & & $26(74.3)$ & $9(25.7)$ & \\
\hline \multicolumn{10}{|l|}{ Staging group $(\mathrm{n}=80)$} \\
\hline Low (1A, IIA, IIB) & $30(90.9)$ & $3(9.1)$ & 0.687 & $24(72.7)$ & $9(27.3)$ & 0.256 & $22(66.7)$ & $11(33.3)$ & 0.446 \\
\hline High (III, IV) & $44(93.6)$ & $3(6.4)$ & & $40(85.1)$ & $7(14.9)$ & & $36(76.6)$ & $11(23.4)$ & \\
\hline \multicolumn{10}{|l|}{ Grade $(\mathrm{n}=80)$} \\
\hline Well & $5(100)$ & $0(0)$ & NA & $5(100)$ & $0(0)$ & 0.729 & $3(60)$ & $2(40)$ & 0.041 \\
\hline Moderate & $47(92.2)$ & $4(7.8)$ & & $40(78.4)$ & $11(21.6)$ & & $33(64.7)$ & $18(35.3)$ & \\
\hline High & $22(91.7)$ & $2(8.3)$ & & $19(79.2)$ & $5(20.8)$ & & $22(91.7)$ & $2(8.3)$ & \\
\hline
\end{tabular}

$\mathrm{SD}$, standard deviation; NA, not applicable; P value $\leq 0.05$ is considered significant.

differentiation between PDAC and benign pancreatic tissue. Whereas, specificity and PPV were $100 \%$ for each of the studied panels (Table 3 ).

Among all studied clinico-pathological data, Ki-67-positive PDAC cases only showed statistical significant relation with tumor grade, compared with negative ones. Ki-67 positivity was significantly higher in poorly differentiated PDAC, represented 22 out of 24 cases (91.7\%), compared to well and moderately differentiated tumors, represented 3 out of 5 cases $(60 \%)$ and 33 out of 51 cases $(64.7 \%)$, respectively. In contrast, both Maspin and
CK17 immunostaining were not significantly correlated with all studied clinico-pathological data (Table 4).

\section{Discussion}

Differentiation of PDAC, especially low grades, from benign pancreatic tissues is one of the obstacles that facing cytopathologists in the diagnosis of pancreatic lesions in EUS-FNAC (Furhata et al., 2017). In this study we aimed to providing information about a new combination of ICC antibodies (Maspin, CK17 and Ki-67) as an ancillary study 


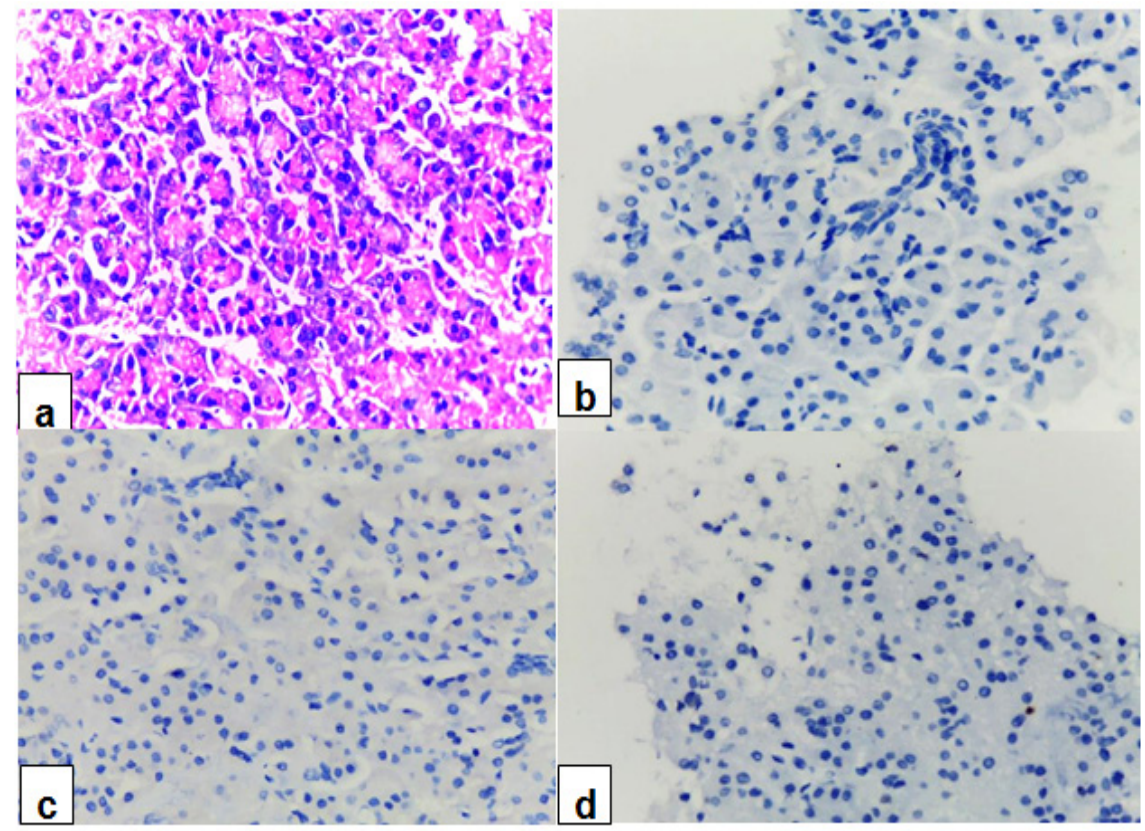

Figure 4. Normal Pancreatic Tissue (Control Group), Aspirated from the Pancreatic Surgically Resected Specimens: (a) H\&E-stained cell block section (x40), (b) Maspin shows negative immunostaining (x40), (c) CK17 shows negative immunostaining (x40) and (d) Ki-67 shows negative immunostaining (x40).

in diagnosis of EUS-FNAC of PDAC. Although many studies evaluated one or two of these markers in diagnosis of PDAC (Yang et al., 2012; Furuhata et al., 2017; AksoyAltinboga et al., 2018), to our best knowledge, we are the first in the literature to test the combination of these three markers together and we are also the first to study the usefulness of CK17 in diagnosing PDAC on cytologic material.

In our study, we found that Maspin, CK17 and Ki-67 were very useful markers in differentiating PDAC from benign pancreatic tissue with a sensitivity of $92.5 \%, 80 \%$ and $72.5 \%$, respectively and the specificity was of $100 \%$ for each marker. Among our control group, no one showed positive staining for any of the studied markers.

In the existing work, the expression of Maspin in PDAC cases was almost similar to the majority of studies in literature $(>/=90 \%$ of cases $)$ and on the same line, all the benign pancreatic tissues were negative for it (Cao et al., 2007; Berardi et al., 2013; Aksoy-Altinboga et al., 2018). Aksoy-Altinboga et al., (2018) reported that using Maspin in the differential diagnosis of PDAC from benign/ reactive pancreatic tissue achieved diagnostic sensitivity and accuracy of $87.5 \%$ and $91.2 \%$ respectively, which were slightly lower than what we recorded, while they recorded higher NPV of $89 \%$, whereas specificity and PPV were similar to our findings (100\%, each).

Regarding the subcellular localization of Maspin immunostaining positivity, there is wide variation in the literature with lack of standardization. For example, Cao et al., (2007) documented that $87 \%$ of cases had only cytoplasmic expression, while the majority of our Maspin positive PDAC cases $(77 \%)$ had nuclear and cytoplasmic immunostaining. Berardi et al., (2013) and Banias et al., (2019) explained that the different position of Maspin inside the cell is related to epigenetic changes. They demonstrated that in malignant tumors, Maspin expression and its subcellular localization influence both tumor behavior and response to chemotherapy. However few studies in literature investigated the significance of subcellular localization of Maspin in PDAC. Cao et al., (2007) proved that nuclear labeling of Maspin in PDAC is associated with better tumor differentiation although it is not associated with a better prognosis. Berardi et al., (2013) showed that nuclear expression of Maspin in PDAC predicts a poor prognosis.

All studies of CK17 in PDAC come in literature were done on surgically resected specimens, with wide variation in positivity rate. Higher figures were noted by Chu et al., (2005), sarbia et al., (2007) and Yang et al., (2012), represented $83 \%, 88 \%$ and $92 \%$ of PDAC cases, respectively. In contrast, CK17 was only found in $60 \%$ of pancreaticobiliary adenocarcinomas (Goldstein and Bassi, 2001). Yang et al., (2012) also found that CK17 was totally negative in normal pancreas.

Few studies investigated the expression of Ki-67 in PDAC, with results reflect usage of variable cutoff points for positivity. Kim et al., (2015) found that Ki-67 was positive in $88.2 \%$ of the PDAC cases, considering $1 \%$ as a cutoff point for positivity. Whereas a lower figure was reported by Jahng et al., (2010) who considered the intense immunostaining for greater than $50 \%$ of population as a cutoff point for positivity. They reported that $41 \%$ of PDAC cases were positive for Ki-67, while all benign cases of pancreatitis were negative.

In the present study, the majority of positive PDAC cases for Maspin and CK17 showed diffuse immunostaining and strong intensity. These results come in concordance with many studies (Chu et al., 2005; Cao et al., 2007; Liu et al., 2012; Aksoy-Altinboga et al., 2018). Whereas study conducted by Goldstein and bassi (2001) revealed that focal and diffuse immunostaining for CK17 are equally distributed among positive PDAC 
cases. Similarly, the majority of our positive PDAC cases for Ki-67 exhibited strong immunostaining intensity, while diffuse immunostaining only represented $22.41 \%$. To the best of our knowledge, no studies in the literature studied the percentage of positive cells and intensity of Ki-67 immunostaining in PDAC.

In our study, for differentiation between PDAC and benign pancreatic tissue, we found that Maspin on its own had the highest sensitivity, NPV and accuracy, when compared with CK17 and Ki-67. Specificity and PPV were $100 \%$ for each marker. We demonstrated that combined use of Maspin, CK17 and Ki-67 as a triple panel with at least one of them is positive, achieved the highest sensitivity, NPV and accuracy of 98.8\%, 96.2\% and $99 \%$ for diagnosing PDAC, when compared to using each two of them separately (alternatively double panels).

Liu et al., (2012) studied 26 markers in the differentiation between PDAC and normal/non-neoplastic pancreatic tissues. In concordance with our study, all PDAC cases (100\%) showed positive staining for Maspin. Whereas CK17 was only positive in $60 \%$ of PDAC cases, which was much lower than we found. Both Maspin and CK17 were negative in all normal/non neoplastic pancreatic tissues. The same findings noticed by Lok et al., (2014) who evaluated immunohistochemical panel in distinction between intrahepatic cholangiocarcinoma and metastatic PDAC on a liver biopsy. CK 17 was only seen in $60 \%$ of cases of PDAC, while all PDAC cases showed positive Maspin immunostaining.

Furhata et al., (2017) considered a higher cutoff point (26\%) for Maspin positivity. They found that Maspin was positive in $40 \%$ of PDAC and Ki-67 was positive in 53 $\%$ of PDAC, while no one of normal pancreatic ductal tissues was positive for both markers.

In correlation between our studied markers and studied clinico-pathological data, the only one was found to have statistical significant relation was $\mathrm{Ki}-67$-positive tumors with tumor grade, compared with negative ones. Kim et al., (2015) similarly found that Ki-67 expression was correlated with pathological grade. They also found a correlation with lymph node, metastasis, and clinical stage. Whereas, Karamitopoulou et al., (2010) reported that Ki-67 labeling index did not show any significant association with tumor grade, $\mathrm{T}$ stage or lymph node status. In contrast, both Maspin and CK17 immunostaining were not significantly associated with all our studied clinico-pathological data. Cao et al., (2007) also noticed that Maspin expression was not correlated with multiple clinico-pathologic variables. To the best of our knowledge, no studies in the literature correlated the significant relationship between CK17 immunostaining and clinicopathological variables in PDAC.

To conclude, Maspin, CK17 and Ki-67 seem to be helpful markers in increasing the accuracy of diagnosing PDAC in EUS-FNAC. Usage of Maspin, CK17 and $\mathrm{Ki}-67$ as a triple test (at least one of them is positive) is a potential ICC panel in the differentiation between PDAC and benign pancreatic tissue. Out of the 3 studied markers, the only one showed statistically significant relation with tumor grade is $\mathrm{Ki}-67$ positive cases, compared with the
Ki-67-negative ones.

\section{Author Contribution Statement}

The authors confirm contribution to the paper as follows: study conception and design: Dr Mona M. Mamdouh, Dr Emad Hamza El-Gemeie, Dr Hussein Okasha, Dr HebatAllah M. Shaaban and Dr Nesreen H. Hafez.; data collection: Dr Mona M. Mamdouh; analysis and interpretation of results: Dr Mona M. Mamdouh, Dr Emad Hamza El-Gemeie, Dr Hussein Okasha, Dr HebatAllah M. Shaaban and Dr Nesreen H. Hafez.; draft manuscript preparation: Dr Mona M. Mamdouh. All authors reviewed the results and approved the final version of the manuscript.

\section{Acknowledgments}

None.

\section{References}

Aksoy-Altinboga A, Baglan T, Umudum H, Ceyhan K (2018). Diagnostic value of S100p, IMP3, Maspin, and pVHL in the differantial diagnosis of pancreatic ductal adenocarcinoma and normal/chronic pancreatitis in fine needle aspiration biopsy. J Cytol, 35, 247-51.

Banias L, Jung I, Gurzu S (2019). Subcellular expression of maspin - from normal tissue to tumor cells. World $J$ Meta-Anal, 7, 142-55.

Berardi R, Morgese F, Onofri A, et al (2013). Role of maspin in cancer. Clin Transl Med, 2, 1-9.

Cao D, Zhang Q, Wu LS, et al (2007). Prognostic significance of maspin in pancreatic ductal adenocarcinoma: tissue microarray analysis of 223 surgically resected cases. Mod Pathol, 20, 570-8.

Cong L, Liu Q, Zhang R, et al (2018). Tumor size classifcation of the 8th edition of TNM staging system is superior to that of the 7th edition in predicting the survival outcome of pancreatic cancer patients after radical resection and adjuvant chemotherapy. Sci Rep, 8, 10383.

Chu PG, Schwarz RE, Lau SK, Yen Y, Weiss LM (2005). Immunohistochemical staining in the diagnosis of pancreatobiliary and ampulla of Vater adenocarcinoma: application of CDX2, CK17, MUC1, and MUC2. Am J Clin Pathol, 29, 359-67.

Dim DC, Jiang F, Qiu Q, et al (2014). The usefulness of S100P, mesothelin, fascin, prostate stem cell antigen, and 14-3-3 sigma in diagnosing pancreatic adenocarcinoma in cytological specimens obtained by endoscopic ultrasound guided fine-needle aspiration. Diagn Cytopathol, 42, 193-9.

Dimastromatteo J, Brentnall T, Kelly KA (2017). Imaging in pancreatic disease. Nat Rev Gastroenterol Hepatol, 14, 97-109.

Foucher ED, Ghigo C, Chouaib S, et al (2018). Pancreatic ductal adenocarcinoma: a strong imbalance of good and bad immunological cops in the tumor microenvironment. Front Immunol, 9, 1-8.

Furuhata A, Minamiguchi S, Shirahase H, et al (2017). Immunohistochemical antibody panel for the differential diagnosis of pancreatic ductal carcinoma from gastrointestinal contamination and benign pancreatic duct epithelium in endoscopic ultrasound-guided fine-needle aspiration. Pancreas, 46, 531-8.

Goldstein NS, Bassi D (2001). Cytokeratins 7, 17, and 20 reactivity 
in pancreatic and ampulla of vater adenocarcinomas: percentage of positivity and distribution is affected by the cut-point threshold. Am J Clin Pathol, 115, 695-702.

He J, Ahuja N, Makary MA, et al (2014). 2564 resected periampullary adenocarcinomas at a single institution: trends over three decades. $H p b, \mathbf{1 6}, 83-90$.

Hirai K, Koizumi K, Haraguchi S, et al (2005). Prognostic significance of the tumor suppressor gene maspin in non-small cell lung cancer. Ann Thorac Surg, 79, 248-53.

Jahng AW, Reicher S, Chung D, et al (2010). Staining for p53 and Ki-67 increases the sensitivity of EUS-FNA to detect pancreatic malignancy. World J Gastrointest Endosc, 2, 362-8.

Karamitopoulou E, Zlobec I, Tornillo L, et al (2010). Differential cell cycle and proliferation marker expression in ductal pancreatic adenocarcinoma and pancreatic intraepithelial neoplasia (PanIN). Pathology, 42, 229-34.

Kim H, Park CY, Lee JH, et al (2015). Ki-67 and p53 expression as a predictive marker for early postoperative recurrence in pancreatic head cancer. Ann Surg Treat Res, 88, 200.

Klein WM, Hruban RH, Klein-Szanto AJ, Wilentz RE (2002). Direct correlation between proliferative activity and dysplasia in pancreatic intraepithelial neoplasia (PanIN): additional evidence for a recently proposed model of progression. Mod Pathol, 15, 441-7.

Kudo T, Kawakami H, Kuwatani M, et al (2014). Influence of the safety and diagnostic accuracy of preoperative endoscopic ultrasound-guided fine-needle aspiration for resectable pancreatic cancer on clinical performance. World J Gastroenterol, 20, 3620-7.

Lin F, Eric Chen Z, Wang HL (2015). Utility of immunohistochemistry in the pancreatobiliary tract. Arch Pathol Lab Med, 139, 24-38.

Liu H, Shi J, Anandan V, et al (2012). Reevaluation and identification of the best immunohistochemical panel (pVHL, Maspin, S100P, IMP-3) for ductal adenocarcinoma of the pancreas. Arch Pathol Lab Med, 136, 601-9.

Lok T, Chen L, Lin F, Wang HL (2014). Immunohistochemical distinction between intrahepatic cholangiocarcinoma and pancreatic ductal adenocarcinoma. Hum Path, 45, 394-400.

Pitman MB, Centeno BA, Ali SZ, et al (2014). Standardized terminology and nomenclature for pancreatobiliary cytology: the Papanicolaou Society of Cytopathology guidelines. Diagn Cytopathol, 42, 338-50.

Reid MD, Centeno BA (2014). Ancillary studies, including immunohistochemistry and molecular studies, in pancreatic cytology. Surg Pathol Clin, 7, 1-34.

Sarbia M, Fritze F, Geddert H, et al (2007). Differentiation between pancreaticobiliary and upper gastrointestinal adenocarcinomas: is analysis of cytokeratin 17 expression helpful?. Am J Clin Pathol, 128, 255-9.

Stuart-Harris R, Caldas C, Pinder SE, Pharoah P (2008). Proliferation markers and survival in early breast cancer: a systematic review and meta-analysis of 85 studies in 32,825 patients. Breast J, 17, 323-34.

Umekita Y, Ohi Y, Sagara Y, Yoshida H (2002). Expression of maspin predicts poor prognosis in breast-cancer patients. Int $J$ Cancer, 100, 452-5.

Umekita Y, Souda M, Yoshida H (2006). Expression of maspin in colorectal cancer. In Vivo, 20, 797-800.

Viale G, Giobbie-Hurder A, Regan MM, et al (2008). Prognostic and predictive value of centrally reviewed Ki-67 labeling index in postmenopausal women with endocrine-responsive breast cancer: results from Breast International Group Trial 1-98 comparing adjuvant tamoxifen with letrozole. J Clin Oncol, 26, 5569-75

Yang HS, Tamayo R, Almonte M, et al (2012). Clinical significance of MUC1, MUC2 and CK17 expression patterns for diagnosis of pancreatobiliaryarcinoma. Biotech Histochem, 87, 126-32.

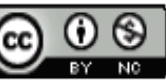

This work is licensed under a Creative Commons AttributionNon Commercial 4.0 International License. 